\title{
Francis Dreyer, Éclairer la mer. Les optiques de phares et leurs constructeurs
}

Rennes, Presses universitaires de Rennes, coll. Arts et Sociétés, 2016

\section{Vincent Guigueno}

\section{(2) OpenEdition}

\section{Journals}

Édition électronique

URL : http://journals.openedition.org/artefact/912

DOI : $10.4000 /$ artefact.912

ISSN : 2606-9245

Éditeur :

Association Artefact. Techniques histoire et sciences humaines, Presses universitaires du Midi

Édition imprimée

Pagination : 214-215

ISBN : 978-2-7535-7305-5

ISSN : 2273-0753

Référence électronique

Vincent Guigueno, « Francis Dreyer, Éclairer la mer. Les optiques de phares et leurs constructeurs », Artefact [En ligne], 6 | 2017, mis en ligne le 31 mai 2018, consulté le 24 septembre 2020. URL : http:// journals.openedition.org/artefact/912 ; DOI : https://doi.org/10.4000/artefact.912

Ce document a été généré automatiquement le 24 septembre 2020.

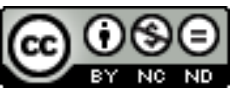

Artefact, Techniques, histoire et sciences humaines est mise à disposition selon les termes de la Licence Creative Commons Attribution - Pas d'Utilisation Commerciale - Pas de Modification 4.0 International. 


\title{
Francis Dreyer, Éclairer la mer. Les optiques de phares et leurs constructeurs
}

Rennes, Presses universitaires de Rennes, coll. Arts et Sociétés, 2016

\author{
Vincent Guigueno
}

\section{RÉFÉRENCE}

Francis Dreyer, Éclairer la mer. Les optiques de phares et leurs constructeurs, Rennes, Presses universitaires de Rennes, coll. Arts et Sociétés, 2016, 312 p.

1 L'ouvrage proposé par les Presses universitaires de Rennes est la publication d'une thèse consacrée aux constructeurs d'optiques soutenue par Francis Dreyer, en 2008, à l'université Paris 4 Sorbonne, sous la direction de Pascal Griset. L'auteur, historien et conservateur du patrimoine, a ensuite poursuivi sa carrière dans le domaine du patrimoine industriel, dans la Nièvre notamment. C'est un expert reconnu à qui les ministères de l'Écologie et de la Culture confient des missions d'inventaire des patrimoines mobiliers et immobiliers des phares ${ }^{1}$. La thèse de Francis Dreyer était la quatrième consacrée aux phares, après le travail fondateur de Jean-Christophe Fichou (1996), publié par le Chasse-Marée en $1999^{2}$, la thèse de Vincent Guigueno consacrée au service des phares (1999), publiée aux Presses universitaires de Rennes en $2001^{3}$, et celle inédite de Marie De Zaedyt (2005), dont seule la partie «coloniale » était novatrice. Francis Dreyer et Jean-Christophe Fichou ont également publié de conserve un premier inventaire des phares de France 4 .

2 Cette production scientifique, sans méconnaître la dimension industrielle de l'histoire des phares, avait comme "personnages» principaux les fonctionnaires de l'administration d'État: ingénieurs des Ponts, savants, gardiens de phares. En mobilisant des sources souvent inédites, Francis Dreyer propose un contre-champ avec cette histoire des entreprises sans qui la politique publique d'éclairage des côtes serait 
restée un projet sans lendemain. Construit en cinq chapitres chronologiques, allant du milieu du XVIII ${ }^{e}$ siècle à la fin du $\mathrm{Xx}^{\mathrm{e}}$, le livre est composé à partir de solides biographies et monographies des acteurs de l'éclairage des mers. Dans les années 1760, l'entrepreneur en éclairage Tourtille-Sangrain fait adopter l'éclairage à l'huile dans les rares tours à feu françaises, malgré l'hostilité des marins qui préfèrent les rustiques foyers de charbon, une durée rare en temps de guerre. L'opticien François Soleil, le bien-nommé, à qui Fresnel doit la construction de ses premiers appareils dans les années 1820 , contribue à une révolution technologique qui donne naissance à une industrie nationale conquérante: les constructeurs d'optiques. Née dans les ateliers d'horlogerie ou d'optique scientifique, l'industrie des phares se structure au mitan du $\mathrm{XIX}^{\mathrm{e}}$ siècle autour de firmes puissantes: Sautter, Lepaute, Barbier, Benard et Turenne (BBT). Un long chapitre est consacré à cette dernière, grâce à des sources inédites recueillies auprès d'anciens cadres de l'entreprise ${ }^{5}$.

Cette approche a de nombreuses vertus : elle relativise la « révolution » des lentilles de Fresnel en montrant que celle-ci bénéficie des rapprochements entre savants, marins et ingénieurs dès la fin de l'Ancien Régime. Elle donne également d'emblée à l'histoire des phares une dimension internationale, puisque les industriels français devinrent les fournisseurs des colonies françaises et de nombreux pays maritimes. La vente des optiques, longtemps construites à Paris, s'inscrit en effet dans l'histoire d'un marché mondial, dont les acteurs sont associés à la normalisation des règles de balisage, en marge des expositions universelles et des congrès de la navigation.

4 Le texte publié présente de salutaires simplifications par rapport à la thèse de 2008, mais aussi d'importants apports, en particulier sur la concurrence de firmes étrangères (l'Anglais Chance Brothers, l'Allemand Pintsch, le Suédois Dalen) subie par les constructeurs français. Le texte est illustré par une iconographie originale de grande qualité rassemblée par l'auteur et complété par de précieuses annexes (tableaux, généalogies, cartes). Il convient ici de souligner le travail éditorial des Presses universitaires de Rennes, dans cette collection dirigée par Jean-Yves Andrieux et Guillaume Glorieux, qui réussit une publication de référence alliant rigueur scientifique et esthétique, l'iconographie du sujet le méritant. En revanche, le titre, volontairement plus accrocheur, n'est ni clair ni convaincant. La bibliographie aurait mérité un "toilettage ", en particulier dans les publications françaises et étrangères les plus contemporaines. On citera, par exemple, les travaux de l'économiste Élodie Bertrand (laboratoire PHARE, EA 7418, université Paris 1 Panthéon-Sorbonne) sur le financement des phares à l'époque moderne, en France et en Angleterre. Malgré ces réserves mineures, le livre de Francis Dreyer vient heureusement compléter la bibliothèque des phares, en espérant que des travaux futurs viennent s'intéresser à une histoire plus intégrée de la sécurité de la navigation. 


\section{NOTES}

1. Lire sa contribution à l'inventaire des phares de France dans Vincent GUIGUENO, «Les phares des côtes de France : reconnaissance d'un patrimoine ", Patrimoines, $n^{\circ} 12,2016$, p. 34-41.

2. Jean-Christophe FICHOU (dir.), Phares: histoire du balisage et de l'éclairage des côtes de France, Douarnenez, Le Chasse-marée, 1999.

3. Vincent GUIGUENO, Au service des phares. La signalisation maritime en France, $\mathrm{XIX}^{e}-\mathrm{XX}{ }^{e}$ siècle, Rennes, PUR, 2001.

4. Francis DREYER et Jean-Christophe FICHOU, L'histoire de tous les phares de France, Rennes, OuestFrance Édilarge, 2005.

5. Philippe BÉNARD, Une aventure industrielle française BBT, 1862-1984 - De l'optique des phares maritimes à l'optique spatiale, Paris, Éditions du Palio, 2016.

\section{AUTEURS}

\section{VINCENT GUIGUENO}

Musée national de la Marine 